\title{
Xor-Magic Graphs
}

\author{
Jacob A. Siehler \\ Gustavus Adolphus College \\ jsiehler@gustavus.edu
}

\begin{abstract}
A connected graph on $2^{n}$ vertices is defined to be xor-magic if the vertices can be labeled with distinct $n$-bit binary numbers in such a way that the label at each vertex is equal to the bitwise xor of the labels on the adjacent vertices. We show that there is at least one 3-regular xor-magic graph on $2^{n}$ vertices for every $n \geqslant 2$. We classify the 3-regular xor-magic graphs on 8 and 16 vertices, and give multiple examples of 3-regular xor-magic graphs on 32 vertices, including the well-known Dyck graph.
\end{abstract}

Keywords: cubic graph, regular graph, graph theory, combinatorics, linear algebra, binary, xor.

\section{A special labeling of the cube}

The vertices of the cube can be labeled using all eight 3-bit binary numbers in such a way that the bitwise xor of each vertex with its three neighbors is zero. Figure shows such a labeling. The xor condition is satisfied at the back lower right vertex because

$$
111 \oplus 011 \oplus 010 \oplus 110=000,
$$

and you can see that the condition is satisfied at the remaining vertices as well. Other such labelings can be obtained by symmetries of the cube. If you regard a 3-bit binary number as a vector in $\mathbb{F}_{2}^{3}$, then the xor operation is just vector addition, and so you can obtain further labelings of the cube by applying any element of $G L_{3}\left(\mathbb{F}_{2}\right)$ to all of the labels. But how special is the graph of the cube, that it admits any such labeling at all?

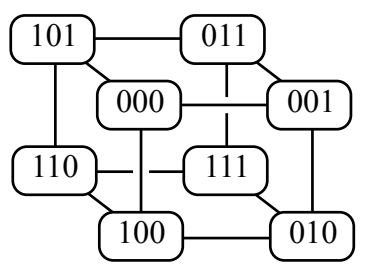

Figure 1: Xor-magic cube. 
Let us define a graph $G$ to be xor-magic (of order $n$ ) if $G$ is connected, has $2^{n}$ vertices, and the vertices of $G$ can be labeled with the distinct $n$-bit binary numbers in such a way that the bitwise xor of every vertex with its three neighbors is zero. Equivalently, if we view an $n$-bit number as a vector in $\mathbb{F}_{2}^{n}$, the magic condition is that all $2^{n}$ vectors are used, and the sum of every vertex with its three neighbors is the zero vector.

Although the xor-magic definition makes sense for any graph on $2^{n}$ vertices, this note will focus on 3-regular graphs. This is partly because the xor-magic property of the cube was discovered in the analysis of a tile-sliding game which can only be played on 3-regular graphs [4. It is also partly just because regularity, with a low vertex degree, makes the graphs more appealing as puzzles, when they are to be worked out by hand.

We will show that 3-regular, xor-magic graphs of every order exist (Theorem 1). We also give necessary conditions for a graph to be xor-magic (Theorem 2), and, with computer assistance, show that 3-regular, xor-magic graphs of small order are rare. Finally, we offer an order 5 xor-magic graph without giving a labeling, for the reader who would like to work it out as a puzzle.

\section{An infinite family}

The complete graph on four vertices is a 3-regular, xor-magic graph of order 2 , and the cube seen above is order 3 . These can be seen as the first two in an infinite family, constructed inductively in a manner reminiscent of the reflected binary Gray code.

Theorem 1. There is a 3-regular, xor-magic graph of order $n$ for every $n \geqslant 2$.

Proof. We begin with a different drawing of the cube graph, shown in Figure 2. The edges extending off the right end are meant to connect to the corresponding edges on the left end, as if the graph were wrapped around a cylinder.

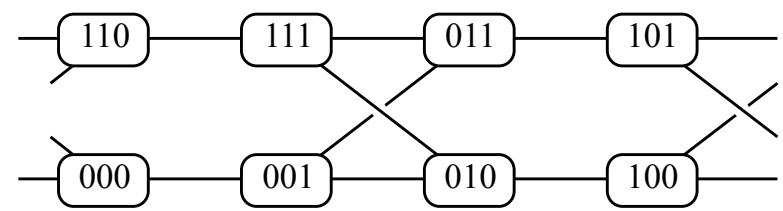

Figure 2: Cube as "crossed prism".

To make an order 4 graph,

1. Make two copies of the order 3 graph. In one copy, append a 0 at the left of every label, and in the second copy, append a 1 at the left. This generates all possible four-bit labels.

2. Glue the two copies together, side by side (Figure 3). 


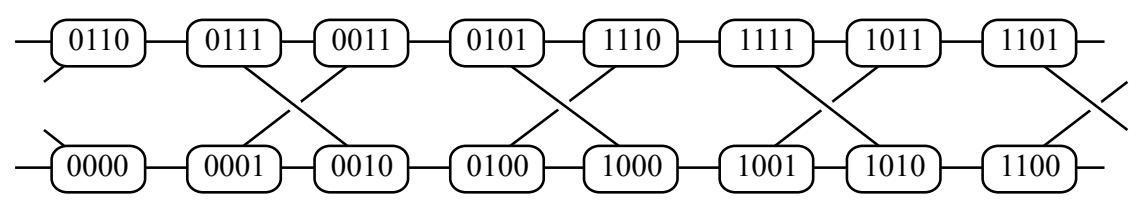

Figure 3: Two copies of the cube, glued together.

The resulting graph is clearly three-regular. At every vertex, the three rightmost bits satisfy the xor condition since they are copied directly from the cube. Any vertex not involved in the gluing has the same leftmost bit as all its neighbors, so the xor condition is satisfied for that bit as well. Any vertex which is on the gluing boundary has one neighbor with the same leftmost bit, and two neighbors with the opposite leftmost bit, so once again, the xor condition is satisfied. We have constructed an order 4 xor-magic graph.

Now, two copies of the order 4 graph can be extended to 5 bits and placed side by side to form an order 5 xor-magic graph, and so on.

\section{Necessary conditions}

The next theorem provides simple calculations that can be used to show that many graphs are not xor-magic. Let $G$ be a graph with vertices numbered 1 through $2^{n}$. Let $M$ be the $2^{n} \times 2^{n}$ matrix with

$$
m_{i j}= \begin{cases}1, & \text { if } i=j \text { or if vertices } i \text { and } j \text { are adjacent in } G \\ 0, & \text { otherwise }\end{cases}
$$

In other words, $M$ is the adjacency matrix of $G$, together with 1 's on the main diagonal. An element in the nullspace of $M$ over $\mathbb{F}_{2}$ is an assignment of single bits to the vertices of $G$ in such a way that the xor condition is satisfied at every vertex. Since vectors are added componentwise, an $n$-bit labeling of $G$ is equivalent to a choice of $n$ vectors in the nullspace of $M$. The first vector gives the first bit in each label; the second vector gives the second bit; and so on.

Let $y_{1}, y_{2}, \ldots, y_{k}$ be any basis for the nullspace of $M$ over $\mathbb{F}_{2}$ (so, $k$ denotes the nullity of $M)$. Finally, let $Y$ be the $2^{n} \times k$ matrix having the $y_{i}$ 's as columns.

Theorem 2. If $G$ is xor-magic, then

(Test 1) $k \geqslant n$, and

(Test 2) The rows of $Y$ are all distinct.

Test 2 is particularly powerful because it applies to any basis for the nullspace.

Proof. Suppose that $G$ is xor-magic. Let $S$ be the $2^{n} \times n$ binary matrix in which the $i$-th row contains the bits in the label of vertex $i$.

For Test 1: Certainly $S$ contains $n$ linearly independent rows (for example, the rows containing a single 1 ). Thus, the rank of $S$ is $n$, and the $n$ column vectors of $S$ are linearly independent. But each of those column vectors is in the nullspace of $M$, so the nullity $k$ is at least $n$. 
For Test 2: Let $Y$ be a matrix whose columns are a basis for the nullspace of $M$, as described above. Since each column of $S$ is in the nullspace of $M$, it can be expressed as a linear combination of the columns of $Y$. Hence, there is a $k \times n$ matrix $J$ with $Y J=S$. Since the rows of $S$ are all distinct, the rows of $Y$ must all be distinct as well.

Note: If a graph passes Test 2, then the rows of the $Y$ matrix provide a set of $k$-bit labels which satisfy the xor condition and are all distinct. If $k=n$, then the graph is xor-magic. However, if $k>n$, there may or may not be a set of distinct $n$-bit labels which satisfy the xor condition.

\section{3-regular graphs on 8 vertices}

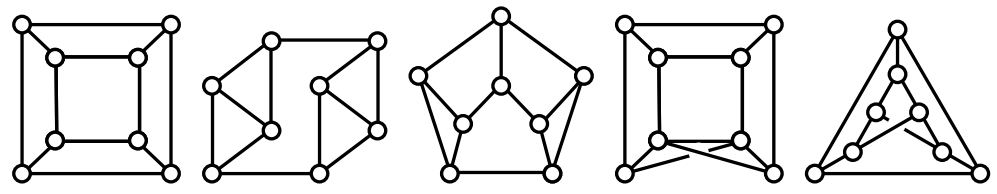

Figure 4: The five connected, 3-regular graphs on 8 vertices.

There are only five connected, 3-regular graphs on 8 vertices (sequence A002851 in the OEIS [5]), and they are shown in Figure 4 .

The nullities of the $M$ matrices for the five graphs are, respectively: 4, 4, 2, 2, and 1 . Thus, all but the first two graphs fail Test 1, and cannot be xor-magic.

The second graph in Figure 4 has sufficient nullity to pass Test 1. This graph's $M$ matrix (with respect to the vertex numbering shown at left) is

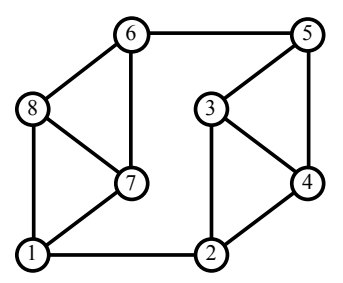

$$
M=\left(\begin{array}{llllllll}
1 & 1 & 0 & 0 & 0 & 0 & 1 & 1 \\
1 & 1 & 1 & 1 & 0 & 0 & 0 & 0 \\
0 & 1 & 1 & 1 & 1 & 0 & 0 & 0 \\
0 & 1 & 1 & 1 & 1 & 0 & 0 & 0 \\
0 & 0 & 1 & 1 & 1 & 1 & 0 & 0 \\
0 & 0 & 0 & 0 & 1 & 1 & 1 & 1 \\
1 & 0 & 0 & 0 & 0 & 1 & 1 & 1 \\
1 & 0 & 0 & 0 & 0 & 1 & 1 & 1
\end{array}\right)
$$

from which a basis for the nullspace of $M$ can be computed. One such basis is given by the columns of the $Y$ matrix here:

$$
Y=\left(\begin{array}{llll}
1 & 1 & 1 & 0 \\
0 & 0 & 1 & 0 \\
1 & 1 & 0 & 1 \\
0 & 0 & 0 & 1 \\
1 & 1 & 1 & 0 \\
0 & 0 & 1 & 0 \\
0 & 1 & 0 & 0 \\
1 & 0 & 0 & 0
\end{array}\right)
$$


Since this $Y$ matrix has duplicate rows, the graph cannot be xor-magic. Thus, the cube is the only 3 -regular, xor-magic graph on 8 vertices.

\section{3-regular graphs on 16 vertices}

There are 4,060 connected, 3-regular graphs on 16 vertices (see [5], or [2] for details of the calculation), and there are multiple sites on the internet where one can download a data file containing all of them. One such site is House of Graphs (https://hog.grinvin.org/Cubic), which is introduced in [1].

With the hard work of enumeration already done, it is easy to have a computer algebra system do the necessary nullspace calculations to establish the following:

Theorem 3. There are only two connected 3-regular graphs on 16 vertices which pass both tests from Theorem 2 .

For example, in Mathematica, this can be done as follows, where the cub16.g6 file comes from https://hog.grinvin.org/Cubic:

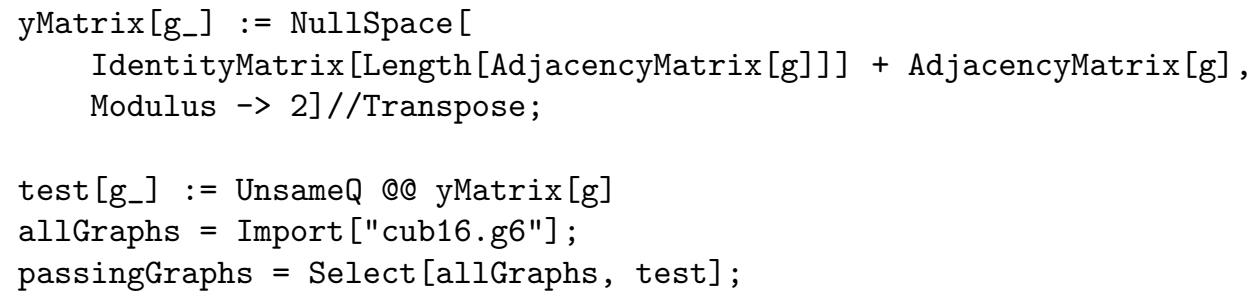

One of them, of course, is the crossed prism constructed in Theorem 1 . The other, which we shall denote $G_{1}$, is shown in Figure 5 . The nullity of the $M$

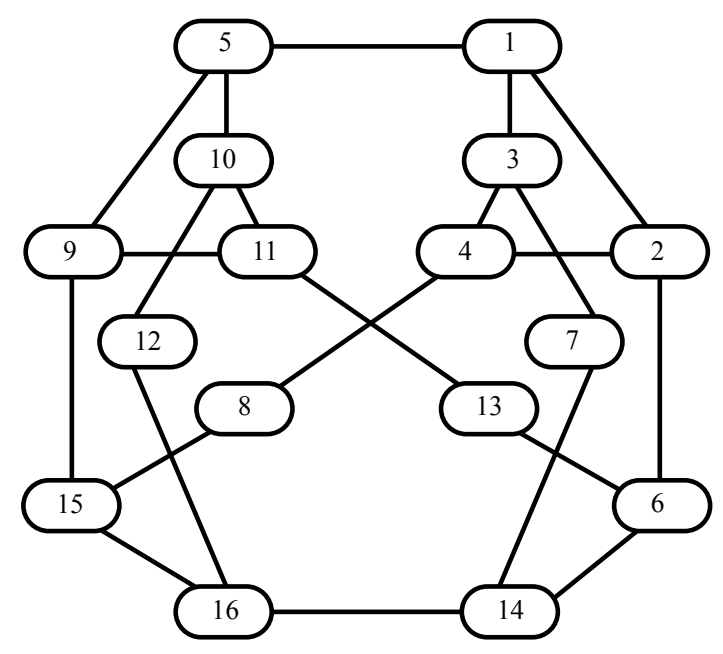

Figure 5: $G_{1}$ passes both tests in Theorem 2 .

matrix for $G_{1}$ is 6 , and computing a basis for the nullspace gives a $Y$ matrix with distinct rows. However, this only goes to demonstrate that the necessary conditions of Theorem 2 are not sufficient: 
Theorem 4. $G_{1}$ is not xor-magic.

The crossed prism is thus the only 3-regular, xor-magic graph of order 4. Before we begin the proof, let us note two generally useful transformations. Suppose that a graph $G$ has a set of $n$-bit labels which satisfy the xor-magic condition.

1. Applying any invertible linear transformation in $G L_{n}\left(\mathbb{F}_{2}\right)$ to all the labels preserves the xor-magic property.

2. If every vertex in $G$ has odd degree, then adding a constant $n$-bit vector to every label of $G$ preserves the xor-magic property.

Proof of Theorem 4. Throughout the proof, we will refer to the numbering of the vertices shown in Figure 5 . Suppose that $G_{1}$ has an xor-magic labeling, and let $v_{i}$ denote the 4-bit label on vertex number $i$. By adding $v_{1}$ to every label in the graph, we can assume that $v_{1}=0000$.

If $v_{1}, v_{2}, v_{3}$, and $v_{4}$ are known, then $v_{5}$ through $v_{8}$ are determined by the xor condition - in fact, they are linear combinations of $v_{1}$ through $v_{4}$. Since all eight of these labels must be different, and we have normalized $v_{1}$ to $0000, v_{2}$, $v_{3}$ and $v_{4}$ must be linearly independent. By applying a linear transformation, we can change those to any three linearly independent labels we like. After this transformation, we may assume that the first eight vertices are labeled as follows:

$$
\begin{array}{llll}
v_{1}=0000 & v_{2}=0001 & v_{3}=0010 & v_{4}=0100 \\
v_{5}=0011 & v_{6}=0101 & v_{7}=0110 & v_{8}=0111
\end{array}
$$

Now, whatever $v_{9}$ is, it begins with a 1 , so it is linearly independent from the labels at vertices 2, 3, and 4, and we can apply a linear transformation to assume that $v_{9}=1000$ (while preserving the labels on the first eight vertices). It then follows that $v_{10}=1011$.

At this point, the xor condition requires $v_{11} \oplus v_{12}=v_{5} \oplus v_{10}=1000$. But this is impossible since $v_{11}$ and $v_{12}$ must both have a 1 in the leftmost position. As noted before Theorem 4 , the $M$ matrix for $G_{1}$ has nullity 6 , and since it passes Test 2, its $Y$ matrix provides a set of distinct 6 -bit labels which satisfy the xor condition. In fact, one can improve this to give $G_{1}$ a set of distinct 5-bit labels which satisfy the xor condition (this is not too difficult, and is left as an exercise for the interested reader). $G_{1}$ is not magic, but it is very close. In general, for a graph which passes both tests from Theorem 2 with $k>n$, the author does not know a way to determine the minimum number of bits which suffice to give the vertices distinct labels which satisfy the xor condition.

\section{More than just crossed prisms}

At this point, one might guess that the crossed prisms of each order are the only 3-regular xor-magic graphs, but that is not so. An exhaustive search of 32 -vertex graphs is not feasible. However, searching among the most symmetric 3 -regular graphs is surprisingly fruitful. There are just ten connected, vertextransitive cubic graphs on 32 vertices (A032355 in [6]). Vertex-transitive means that, for any two vertices $u$ and $v$ in the graph, there is a graph automorphism 
which carries $u$ to $v$ - an extra-strong regularity property. Among the ten are four xor-magic graphs: the crossed prism, the Dyck graph, and two others. Figures 6 and 7 show the show magic labelings of the Dyck graph and one of the others. Figure 8 is the remaining graph, given without a labeling for readers who might enjoy working out a solution for themselves, whether computer-assisted or entirely by hand.

\section{Final Remarks}

The xor-magic condition introduced in this note may seem unrelated to the old recreational mathematics standby of magic squares. However, Conway, Norton, and Ryba 3 prove that when the numbers $0-15$ are placed into a traditional $4 \times 4$ magic square, the numbers in each row and each column xor to zero; they call this the Nimm0 property. The present article is inspired by their exposition. With regard to 3-regular, xor-magic graphs, it would be interesting to know if other infinite families with simple constructions like the crossed prisms might exist - perhaps some or all of the 32-vertex graphs in Figures 60 represent the beginning of some such family. We have already acknowledged that the focus on 3-regular graphs in this article is somewhat arbitrary, and many other xor-magic constructions may be waiting to be found among other classes of graphs.

Most interesting of all would be better theory to determine the minimum number of bits required to give a graph a set of distinct labels which satisfies the xor condition. An upper bound is given by Theorem 2 , but the 5 -bit labeling of $G_{1}$ shows that it is not sharp. Finding good theorems or algorithms to lower this bound should be challenging work for the future.

\section{References}

[1] Brinkmann, G., Coolsaet, K., Goedgebeur, J., Mélot, H. "House of graphs: a database of interesting graphs", Discrete Applied Mathematics, 161(1-2), 311-314, 2013.

[2] Brinkmann, G., Goedgebeur, J., D McKay, B. "Generation of cubic graphs". Discrete Mathematics and Theoretical Computer Science, 13(2), 69-79, 2011.

[3] Conway, J., Norton, S., Ryba, A. "Frenicle's 880 magic squares", The Mathematics of Various Entertaining Subjects: Research in Games, Graphs, Counting, and Complexity, 2, 71, 2017.

[4] Ekenta, O., Han Gil Jang, Siehler, J. "Slide-and-swap permutation groups", Involve, a Journal of Mathematics, 7(1), 41-55, 2013.

[5] N. J. A. Sloane. Online encyclopedia of integer sequences. http://oeis.org/A002851.

[6] N. J. A. Sloane. Online encyclopedia of integer sequences. http://oeis.org/A032355. 


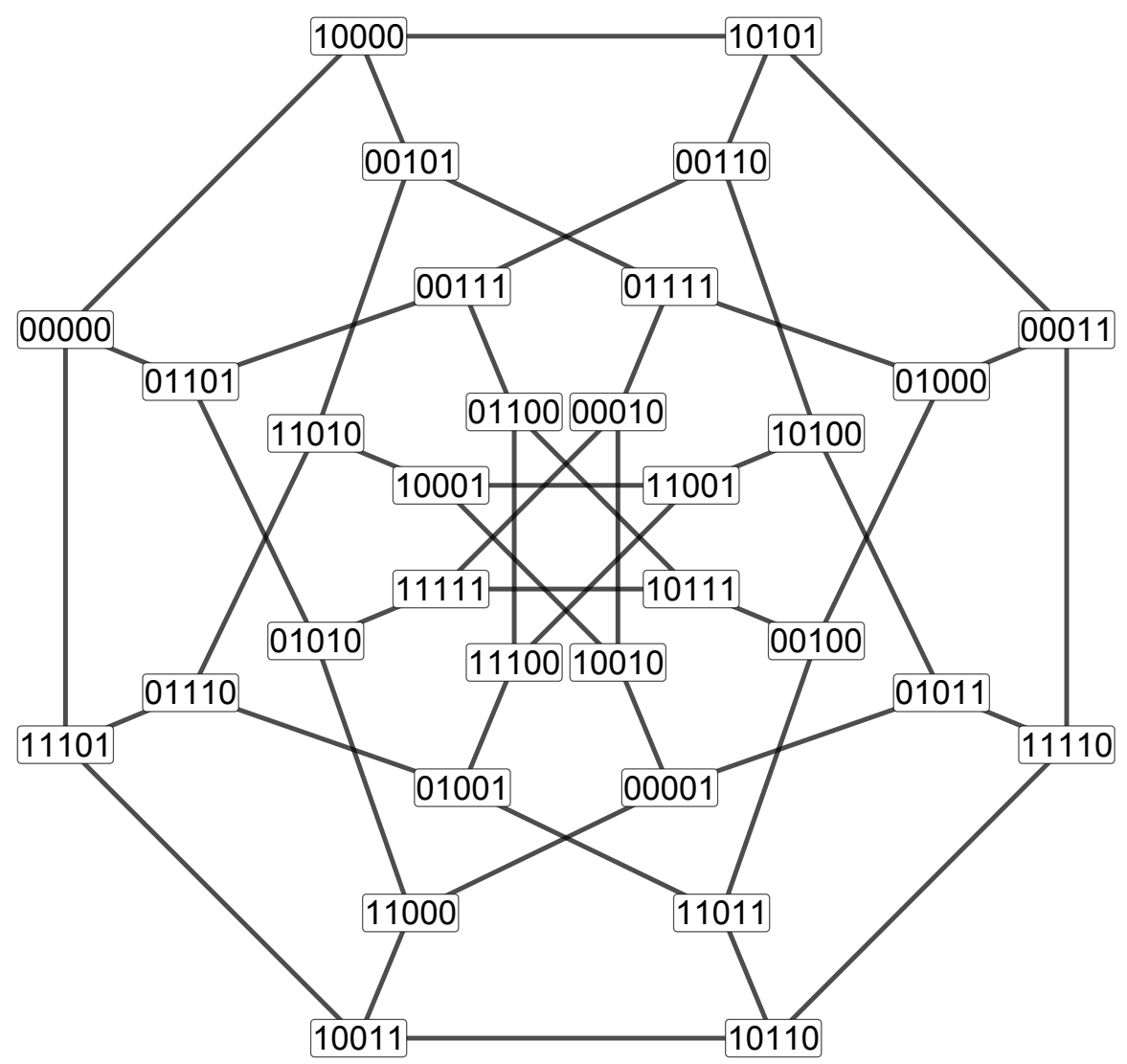

Figure 6: The Dyck graph is xor-magic. 


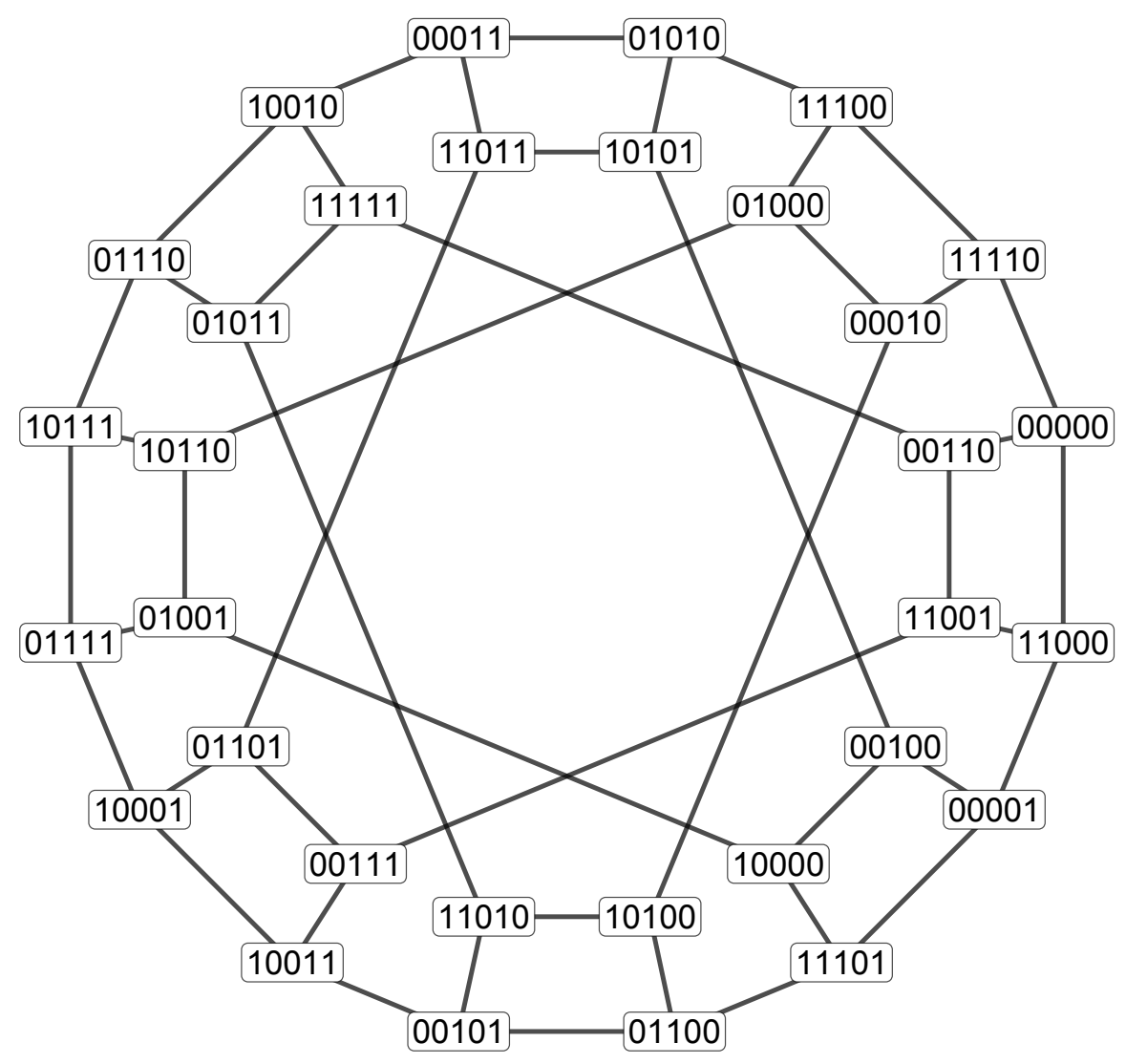

Figure 7: Another vertex-transitive xor-magic graph. 


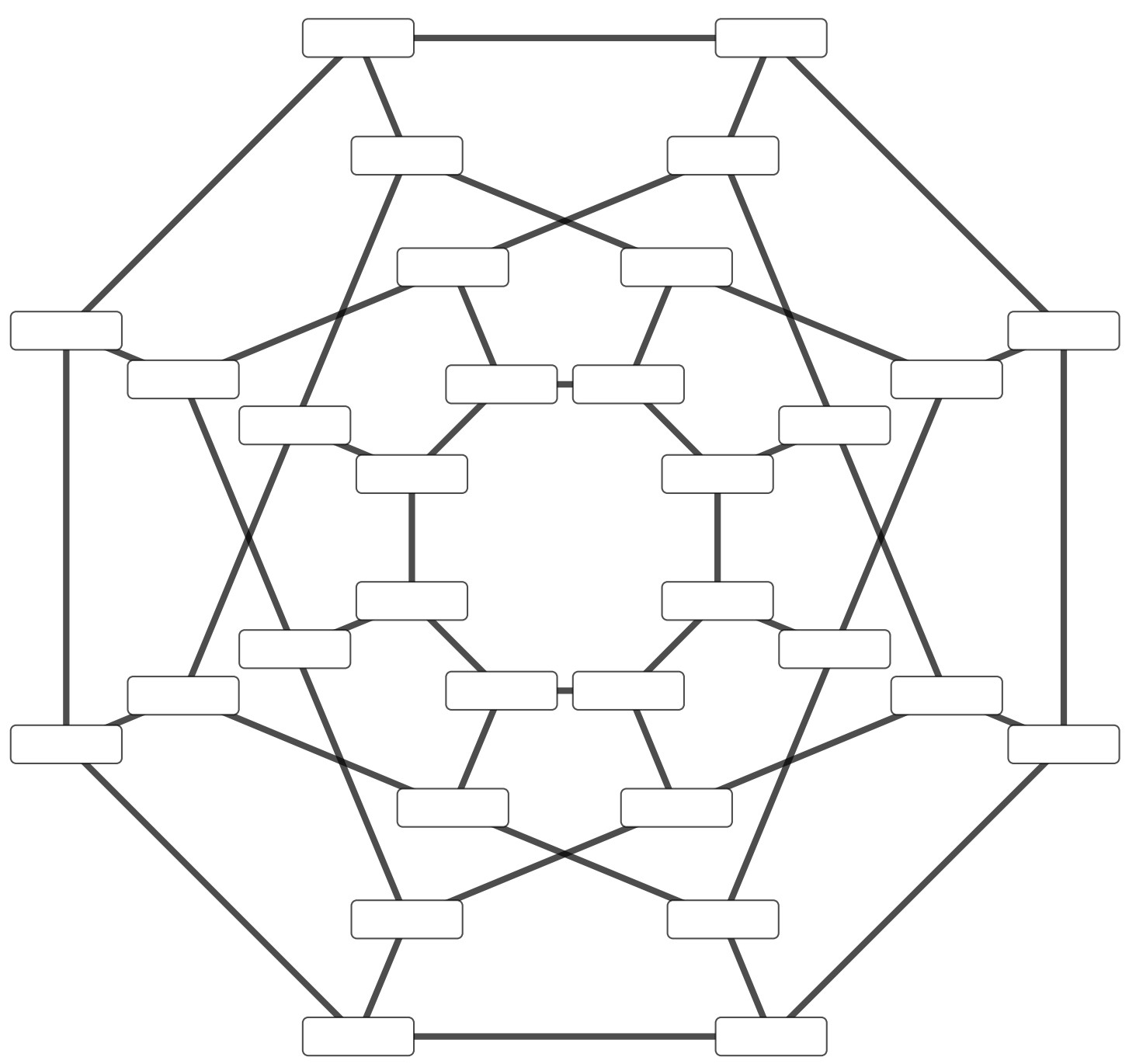

Figure 8: Order 5 xor-magic graph, without its labels. 